\title{
EL FUTURO DE LAS CIUDADES DIGITALES: RETOS, OPORTUNIDADES Y PROSPECTIVAS
}

\section{SMART CITIES: CHALLENGES, OPPORTUNITIES, AND PROSPECTS}

\author{
Piergiorgio Degli Esposti \\ Universidad de Bolonia, Bolonia / Italia \\ pg.degliesposti@unibo.it \\ https://orcid.org/0000-0001-8930-0859
}

Giovanni Ciofalo Sapienza Universidad de Roma, Roma / Italia giovanni.ciofalo@uniroma1.it https://orcid.org/0000-0002-2932-9315

Recibido/Received: 26/04/2020

Modificado/Modified: 28/05/2020

Aceptado/Accepted: 10/06/2020

\section{RESUMEN}

El objetivo de este artículo es definir y analizar el concepto de Smart City (ciudad inteligente) a la luz de las evoluciones tecnológicas que las ciudades contemporáneas incorporan progresivamente y de acuerdo con los principales aportes teóricos en el campo socio-lógico. El impacto de las tecnologías digitales está transformando gradualmente nuestras formas de relacionarnos dentro de varios contextos de referencia, no siendo menos importante la ciudad como lugar de producción y consumo. Vivimos cada vez más contextos híbridos, físicos y digitales en los que se forman caminos en diverso sentido derivados de la coexistencia en dos espacios, caminos que llevan a muchos a interpretar cómo el concepto de realidad en sí mismo se enriquece hoy por las oportunidades que ofrece el mundo digital (y viceversa), que fluyen en la dirección de la realidad aumentada, por un lado, y del brick and click (combinación de físico y virtual), por el otro.

\section{PALABRAS CLAVE}

Sociedad digital; realidad aumentada; prosumidor; TIC; ciudad inteligente.

\section{SUMARIO}

1. El complejo fenómeno de la Ciudad Inteligente . 2. La ciudad inteligente como un sistema sociotécnico. 3. Comparación de la realidad física y la esfera digital. 4. Conclusiones: ¿Qué Ciudad Inteligente es posible? 5. Bibliografía.

\footnotetext{
ABSTRACT

Starting from the definition and the analysis of the smart city notion, this article will introduce the main theoretical sociological approaches upon the topic in object. Digital technologies are increasing transforming social behaviors, social relations, and lately even spatial interaction. The city as a place of production, consumption and prosumption thanks to the digitalization is increasing defined as a hybrid space, a mix of physical and digital con-texts. The article will develop an analysis of the hybridization of the city spaces through a series of case studies.
} 


\section{KEYWORDS}

Digital society; Augmented Reality; Prosumer; ICT; Smart city.

\section{CONTENTS}

1. The complex phenomenon of the Smart City. 2. The Smart City as a socio-technical system. 3. Physical reality and the digital sphere. 4. Conclusions: Which Smart City is possible? References.

\section{EL COMPLEJO FENÓMENO DE LA CIUDAD INTELIGENTE}

En los últimos años del siglo XX, la urbanización y las tecnologías de la información y la comunicación (TIC) se han desarrollado en sincronía y de manera imponente, en particular gracias al avance tecnológico y a un significativo crecimiento económico que ha contribuido a aumentar el bienestar generalmente en los principales centros urbanos. A nivel mundial, los procesos de urbanización masivos se pueden asociar a consecuencias positivas, como el aumento de las oportunidades de empleo, el aumento en el nivel de educación y el aumento en el nivel de vida promedio; así como también a consecuencias negativas como, el aumento del tráfico, la producción de dióxido de carbono y las emisiones de gases de efecto invernadero, y la dificultad para la eliminación de residuos.

Se estima que para el 2050 la población mundial crecerá en un $32 \%$ hasta alcanzar los 9,2 mil millones de habitantes, alimentando el fenómeno de las llamadas "megaciudades", particularmente en África, Asia y América Latina. Cítese, por ejemplo, ciudades como Nueva Delhi, Mumbai o Shanghai (Townsend, 2013). Considerando el Programa de las Naciones Unidas para los Asentamientos Humanos (ONU-Hábitat), y su Nueva Agenda Urbana adoptada en octubre 2016 donde las ciudades consumen actualmente el $75 \%$ de la energía mundial, y más del $60 \%$ de la población mundial vivirá en ciudades, el desarrollo y la difusión de las ciudades inteligentes parecen ser fundamentales para satisfacer las futuras demandas de energía y transporte, principalmente de la población, de manera sostenible y eficiente desde el punto de vista ambiental.

El crecimiento rápido y desorganizado de las ciudades que se desbordan de sus propias fronteras incorporando y globalizando territorios genera una expansión urbana y produce una nueva estética urbana, que se convierte en la expresión de transformaciones económicas, sociales y políticas (Pieretti, Castrignanò 2010). La fragmentación de la ciudad expandida, cada vez más constituida por una red de diferentes islas territoriales, refleja la desarticulación de las clases sociales en las sociedades occidentales (Sartoretti, 2012), en la que el ciudadano se traslada diariamente de una isla territorial a otra, ocupando un radio espacial que conecta varias actividades difusas.

La consecuencia directa del fenómeno de expansión es que el derecho a la participación y apropiación de la ciudad, ya descrito por Lefebrve (1968), se extiende cada vez más, no solo a los residentes, sino también a aquellos que consumen en ella y la atraviesan temporalmente, los llamados city users (usuarios de ciudad), los no residentes que, aprovechando los servicios y oportunidades que ofrece la ciudad, también contribuyen a rediseñar sus lógicas, de acuerdo con sus necesidades específicas.

Paralelamente con el aumento del tamaño de las ciudades se ha incrementado el consumo de energía, el uso de los recursos naturales, la necesidad de una remodelación territorial y una infraestructura adecuada. Asociado a estos problemas estructurales relacionados con la evolución del espacio urbano, un fenómeno muy estudiado por sociólogos y planificadores 
urbanos es el de la llamada gentrification (gentrificación), que describe el proceso de renovación de zonas urbanas degradadas a través de la afluencia de grupos sociales más acomodados: una mejora de la calidad material de un sector, que a menudo obliga al traslado de residentes y negocios tradicionales, con un consecuente cambio radical de la composición socio-cultural de áreas urbanas y una pérdida de autenticidad e identidad. Un ejemplo emblemático de estos procesos es la actual ciudad de San Francisco CA, en la cual los capitalistas de riesgo y los expertos de tecnología están literalmente cambiando el tejido urbano y el espíritu de la ciudad.

El concepto mismo de ciudad inteligente puede ser considerado como el siguiente paso después del fenómeno de gentrificación en el proceso general de regeneración urbana, basado en el principio teórico de que las ciudades deben recuperarse a sí mismas, en un cierto modo "reciclarse" a través de la valorización de sus propios contenidos según una nueva modalidad de uso (Masi, 2016).

La ciudad entendida como un organismo vivo ha generado en el tiempo varias y diferentes metáforas interpretativas, entre estas ameritan mencionarse: la ciudad global (Sassen, 1991), la ciudad líquida (Bauman, 2000), la ciudad sostenible (Beck 2000), la ciudad de los individuos (Castrignanò 2004), la ciudad de las redes (Castells, 2004), la ciudad consumada (Pezzini y Cervelli, 2006; Mazzette y Sgroi, 2007), y la ciudad hospitalaria (Costa, 2008).

Sin embargo, no se trata solamente de una cuestión metafórico-semántica, sino de un cambio epistemológico en el que el concepto de crecimiento inteligente difundido en USA a partir de los años 90 representa una alternativa al concepto de expansión y quiere poner en el centro del debate teórico las necesidades de las personas, las estrategias de gobernanza locales y la importancia de las tecnologías habilitantes.

Es en este escenario socio- cultural, y con una visión tecno-ecologista, que se desarrolla la idea de las ciudades "inteligentes", ciudades capaces de resolver, o al menos prevenir, problemas urbanos con la ayuda de dispositivos y plataformas tecnológicas con miras al mejoramiento de la calidad de vida de sus habitantes, para reducir los costos de gestión de servicios urbanos y con una particular atención de respeto al ambiente. Ciudades basadas en un modelo de desarrollo definido como "crecimiento inteligente", entendido como una reacción conducida por la comunidad para resolver los problemas de la vida urbana cotidiana, como: la congestión del tráfico, la sobrepoblación, la contaminación atmosférica y la pérdida del espacio abierto.

El término "inteligente" es usado en múltiples contextos y presenta una flexibilidad semántica extrema. Justamente esta adaptabilidad es un factor decisivo para la gran acogida en los diferentes campos en los que es utilizado, desde las tecnologías hasta la representación de imaginarios urbanos complejos.

Una polisemia que se refleja también en el concepto de "ciudad inteligente", se refiere tanto a un uso sensato y sostenible de los recursos de una ciudad, como también al desarrollo de instrumentos tecnológicos inteligentes (Bria, 2018). Este concepto es atribuible en un nivel teórico a dos paradigmas dominantes de la contemporaneidad: uno ecológico propuesto por Beck, y uno informativo propuesto por Castells.

La evolución de la definición de ciudad inteligente pasa esencialmente por tres macro fases ubicadas temporalmente desde el año 2000 hasta hoy. En la primera fase, la palabra inteligente se refiere a la ciudad digital, principalmente concentrándose en aspectos hardware, es decir las infraestructuras tecnológicas. En fase intermedia, el énfasis se ubica en la ciudad inteligente como ciudad socialmente inclusiva, por ende, se concentra principalmente en los aspectos de software de la ciudad, el capital social y humano y el nivel 
de participación. La tercera fase evolutiva post 2010 se concentra en las relaciones entre la ciudad y la calidad de vida, buscando un equilibrio entre los componentes hardware infraestructura, tecnología y economía- y los componentes software, es decir, las dinámicas humanas del lugar, de las conexiones y relaciones que se establecen entre los diferentes actores sociales.

Para Boyd Cohen (2016), las ciudades inteligentes son ciudades que utilizan las tecnologías de la información y la comunicación (TIC) junto con internet, para ser más eficientes en el uso de los recursos y que por lo tanto, ahorran dinero y reducen desperdicios, mejoran los servicios y la calidad de la vida de los ciudadanos y disminuyen la contaminación. En este sentido, una ciudad inteligente puede entenderse como una ciudad en la cual las tecnologías de la información y la comunicación son aplicadas para mejorar la calidad de los múltiples procesos en los que se articula la vida humana, sean estos: sociales, políticos, ambientales, culturales y económicos.

Las ciudades inteligentes son entonces conceptualizadas como sistemas sociotécnicos que pueden ser estudiados desde la prospectiva de los Estudios de Ciencia y Tecnología, cuyo cuadro teórico de referencia se basa en la Teoría de la Red de Actores, y en la Teoría de Roles para desarrollar un nuevo marco interpretativo útil para distinguir y describir las dinámicas y las interacciones entre actores humanos y avances tecnológicos (come especificaremos en el siguiente párrafo).

Entre los numerosos aportes teóricos para el desarrollo del concepto de ciudad inteligente, de gran interés es la contribución de Giffinger (2007), a través de la identificación de seis categorías interpretativas: economía inteligente, gente inteligente, gobernanza inteligente, movilidad inteligente, entorno inteligente, vida inteligente.

Seis categorías que evidencian como la participación de los diferentes actores en la ciudad inteligente -el sector público, el mercado y la sociedad civil- tiene como objetivo mejorar la calidad de vida de manera sostenible. La economía inteligente es la condición que se da en presencia de un ambiente que genera una competición económica a través del espíritu de innovación, emprendimiento, mercado laboral flexible, vocación internacional y capacidad de transformación. La gente inteligente está representada por la tasa de especialización y educación de las personas que viven en un determinado contexto urbano, pero también por el nivel y la calidad de las interacciones sociales, del pluralismo cultural, de la apertura mental de los habitantes y del nivel de participación en la vida pública. La gobernanza inteligente está representada por la transparencia administrativa y de gestión de la calidad de los servicios ofrecidos, y por el grado de participación pública en los procesos decisionales.

La eficiencia del sistema de transporte, su conexión con infraestructuras TIC y la accesibilidad a nivel local e internacional constituyen la llamada movilidad inteligente. El entorno inteligente está representado por la gestión sostenible y responsable de los recursos, que a través de procesos de reutilización y reciclaje, protege el ambiente y propicia el abatimiento de la contaminación atmosférica perfeccionando la organización y el control para la eliminación de residuos. Última pero no menos importante es la vida inteligente, es decir la calidad de la vida entendida como fermento cultural, salud de los residentes, seguridad y cohesión social y habitacional.

El concepto de inteligente es entonces interpretado desde un sentido de innovación que, incluso antes de la tecnología, se refiere a una idea, a un pensamiento que se gesta como un embrión de innovación tecnológica. Con esta acepción, una ciudad inteligente no es solo una ciudad que comienza a incorporar en su interior nuevas tecnologías innovativas, sino más bien la puesta en acción por un mayor número de personas, una red comunicativa funcional 
y creativa que permite al ciudadano vivir en un modo distinto o nuevo, una red que no tiene únicamente el objetivo de facilitar acciones de manera sistemática, sino también la sensibilización, el conocimiento y el uso adecuado de los recursos. Como afirma Carlo Ratti (2013), las acepciones o significados para hablar de inteligente se han multiplicado en los últimos años: hogar inteligente, ciudadano inteligente, red inteligente, edificio inteligente, solo por citar algunas. Cada una se diferencia de la otra, sin embargo, comparten la idea de un uso distinto de los medios que facilitan la vida cotidiana, especialmente a través y gracias al uso de las nuevas tecnologías.

Si bien la ciudad inteligente puede ser descrita como única, ésta se desarrolla a través de aplicaciones tecnológicas específicas. En este sentido Manville (2014), propone la distinción entre ciudad inteligente, iniciativas de ciudad inteligente, y proyectos de ciudad inteligente. En las iniciativas de ciudad inteligente diferentes actores trabajan juntos en desarrollar objetivos específicos para crear nuevas modalidades de colaboración, proyectos útiles para el desarrollo de la ciudad inteligente. La óptica de red es el elemento típico de la ciudad inteligente, que es facilitado gracias a las TIC, y que permiten a nivel micro conectar proyectos e iniciativas, y al mismo tiempo mejorar la colaboración y coordinación de proyectos a nivel ciudadano. Este proceso lleva a la desaparición progresiva de los límites entre administradores y ciudadanos, así como entre diseño y uso, proponiendo un enfoque "híbrido" que comprende la colaboración entre actores públicos y sociedad civil, en el que múltiples actores están involucrados en diferentes niveles según lógicas similares análogas a las del prosumerismo (Ritzer, 2014; Degli Esposti, 2015), donde ciudadanos activos negocian nuevas formas de poder con las instituciones (Van de Wijdeven, De Graaf, y Hendriks, 2013).

Las ciudades inteligentes usan la tecnología digital con la intención de alcanzar la sostenibilidad ambiental, servicios públicos de calidad y una infraestructura más eficiente para sus residentes. Además de mitigar la brecha digital y asegurar que todos tengan acceso a los datos digitales a través de redes inalámbricas y dispositivos habilitados para la web, las ciudades inteligentes se están concentrando en tres sectores clave para ayudar a los residentes a mejorar la calidad de la vida urbana: energía, transporte y seguridad.

El desarrollo y la alineación del ambiente inteligente a los vectores principales del funcionamiento del organismo urbano interviniendo sobre los nudos cruciales de la metrópoli, actúan como acelerador de nuevos procesos sociales, provocando una mutación de las trayectorias y de las prioridades de desarrollo de la ciudad visto como un sistema.

\section{LA CIUDAD INTELIGENTE COMO UN SISTEMA SOCIOTÉCNICO}

El objetivo del desarrollo sostenible asociado a la difusión urbana ha sido el centro de la atención de arquitectos y administradores públicos. En el libro "La ciudad jardín del mañana" (1898), el urbanista inglés Ebenezer Howard habla sobre cómo el objetivo de los urbanistas debería ser el de transformar los guetos en áreas residenciales capaces de desarrollar oportunidades y bienestar. Le Corbusier y Etchells en el libro "La ciudad del mañana y su planificación" (1929), proponen la idea de cómo se deben corregir los errores generados por la industrialización planteando ciudades que tengan en su interior siempre más espacios verdes para restablecer una relación equilibrada entre el hombre y la naturaleza.

Las ciudades las hacen las personas que viven en ellas y la tecnología debe integrarse en los contextos y las relaciones sociales, en el comportamiento complejo de las personas que 
responden a tantos deseos diferentes (Martinotti 1993). Bruno Latour describe este proceso como "inscribirse, suscribirse a un proyecto": Existen proyectos en los cuales las personas se han inscrito (por ejemplo, el automóvil privado) y otros en cambio en los que no se inscriben, y por ende no funcionan.

La inteligencia de una ciudad está constituida por una serie de infraestructuras físicas y legislativas que sostienen el desarrollo económico, con miras a garantizar la inclusión social y favorecer la protección ambiental, como resultado de la interacción de características que una ciudad debe poseer, y la aplicación de instrumentos a disposición de ciudadanos y administradores para transformar una ciudad en ciudad inteligente (Eremia, Toma, Sanduleac, 2017).

Ojo, et al. (2014), introducen la conceptualización de una ciudad inteligente entendida como un sistema sociotécnico de sistemas. Hablar de sistema sociotécnico significa considerar la coexistencia del sistema técnico representado por las máquinas y los dispositivos útiles para controlarlo, y del sistema social como forma de organización de las personas, normas y roles sociales presentes en un determinado contexto. La hipótesis se desarrolla gracias a la Teoría del Actor-Red, un enfoque teórico destinado a reconocer la capacidad de acción de las tecnologías e incluirlas a título pleno en el panorama de los actores de la red (Latour, 1992); en contraste, la Teoría de Roles sostiene que los roles son cubiertos por personas, por actores humanos. En el ámbito de la Teoría Actor-Red los actores humanos, así como los tecnológicos, conforman una red heterogénea. En esta red las tecnologías son un agente activo, es decir un actor tecnológico. En una ciudad inteligente, las tecnologías de información y comunicación-TIC se aplican para mejorar la calidad de vida en la ciudad, y están intrínsecamente conectadas a los datos que producen los ciudadanos y los dispositivos en sus diversas interacciones con el sistema de la ciudad. Considérese, por ejemplo, los datos sobre el clima o el tráfico alimentados por sensores y dispositivos GPS presentes en los automóviles o por las calles; o también, los datos sobre la salud, presentes tanto en las bases de datos médicas de los hospitales y centros de tratamiento así como también en los dispositivos fitness; o incluso los datos sobre eventos culturales, manifestaciones deportivas o públicas que ahora han encontrado en el panorama de las plataformas de redes sociales un nuevo escenario para la participación.

Como sostienen, Lupton (2015), Ritzer (2014) y Jenkins (2006), entre otros, actualmente vivimos en una sociedad digital donde las tecnologías de este tipo tienen una gran influencia sobre cada aspecto de la vida cotidiana. La transformación en las relaciones sociales, en las relaciones con las instituciones, en la esfera del consumo, o en la difusión del conocimiento, son solo algunos ejemplos de cómo las personas están modificando sus comportamientos a través del uso de tecnologías digitales. Pensar en términos de sociedad digital (Beer, Burrows 2007) significa problematizar el hecho de que el impacto consecuente de la difusión de herramientas, dispositivos y plataformas en la vida cotidiana, en las instituciones y en los procesos de autoidentificación, también desencadena un cambio en los métodos de investigación y análisis.

La continua interacción entre individuo, dispositivos y plataformas digitales, en otras palabras entre ciudadano y ciudad, produce una masa creciente de información, el llamado big data, que ha generado una "revolución digital" (Ash, Kitchin, Leszczynski, 2018), para las ciencias sociales y para la geografía orientada por datos (Miller, Goodchild, 2014). En este sentido, la misma noción de dato adquiere nuevas valencias interpretativas. El dato y la información no son solamente estadísticos, sino que se transforman de manera fluida en elementos "vivos", por el hecho de que son transportados en internet por múltiples plataformas digitales (Hands 2013). Desde este punto de vista internet genera "datos vivos" 
(Lupton 2015).

La vitalidad de los datos se refiere específicamente, según Lupton (2015), a cuatro subcategorías principales:

1. Datos "sobre" la vida: son todos aquellos datos que alimentan el flujo informativo en la red y que pueden ser referidos a la vida de las personas, en el sentido de que puede ser posible reconstruir la biografía de un sujeto a través del análisis de su feed (alimentación);

2. Datos "con" una vida social: es decir todos aquellos que se alimentan de las interacciones sociales que las personas mantienen entre ellas o con instituciones y estructuras de diferente naturaleza.

3. Impacto de los datos en la vida: se refiere a las consecuencias de los datos transportados por la red sobre las personas, y cómo éstos podrían modificar y transformar progresivamente sus vidas.

4. Datos sobre recursos: información sobre los medios de subsistencia y recursos de distinto tipo que poseen las personas.

A través de estas cuatro categorías de datos, los actores humanos y no humanos (tecnológicos) acogen la ciudad como una red, una infraestructura, una plataforma, un sistema sociotécnico.

Basándose en un análisis del discurso científico sobre las ciudades inteligentes, Meijer y Bolívar (2013) han demostrado que existe una confusión sobre el carácter técnico y social de las ciudades inteligentes. Basan sus ideas en las teorías de las Sciences and Technologies Studies-STS (Estudios de Ciencias y Tecnologías), según las cuales, la tecnología debería entenderse como un agente activo dentro de una red de actores, por ejemplo, una tecnología que permite o inhibe acciones específicas, como el resultado de una verdadero y propio guion. (Akrich, 1992). Un ejemplo de esto es sugerido por Ritzer (2018) cuando describe el sistema de gestión de visitantes en los parques temáticos modernos, particularmente Disneyland y Disney World. Los visitantes llegan al parque atravesando una serie de vías que los conducen desde el parqueadero hasta un tren que los acompaña hacia la entrada del parque. Una vez dentro del parque, se crea una larga fila de personas que se mueven como si fuera una enrome cinta transportadora que los lleva desde una atracción a la otra. Las atracciones y los paseos en sí son casi siempre medios de transporte como: autos, barcos, submarinos, aviones, cohetes o aceras móviles que trasladan a la gente rápidamente dentro y fuera del parque temático. Además, para gestionar las hordas de visitantes que se presentan durante la temporada turística, cuando la espera en la fila se torna larga, el parque ha realizado billetes especiales fastpass de acceso rápido que permiten a los visitantes programar cuando quieren subir a una determinada atracción, ingresando por un acceso de preferencia, con menos personas, y por ende, mucho más rápido. Naturalmente, para preservar la eficiencia hay un límite en el número máximo de fastpass vendibles en un día. En el intento de aumentar todavía más la eficiencia y reducir los tiempos de espera, Disney desde el 2014 ha introducido también el sistema fastpass+, aún más rápido, que permite a los visitantes reservar con anticipación máximo tres atracciones.

Si los parques temáticos representan por una parte un simulacro de la ciudad inteligente contemporánea, algunos sistemas similares se han adoptado en algunas ciudades; se piensa en "VeneziaUnica", la tarjeta para optimizar el flujo de turistas en las naves y principales atracciones turísticas de la ciudad, o la "Oyster" travelcard en la ciudad de Londres. Basado en lógicas análogas, también se considera el "Telepass" para autopistas y parqueaderos, así como todas las plataformas de movilidad compartida como Mobike o Uber, o de alojamiento compartido como AirBnb. En todos estos casos las App y la tarjeta de crédito habilitan o 
excluyen el acceso a un determinado servicio.

El guion se entiende como un conjunto de posibilidades que una determinada tecnología ofrece, escrito por proyectistas o desarrolladores de la misma, y prescribe acciones determinadas por los usuarios; los usuarios por su parte, pueden aceptar (subscribirse), rechazar o modificar el guion, lo que puede llevar al desarrollo de anti-programas, de tal manera que la tecnología sea capaz de influir en la distribución de los roles en la red de los actores, determinando una "geografía de la responsabilidad" (Akrich, 1992). Respecto a la relación entre guion y tecnología es necesario resaltar dos factores clave: la obsolescencia a la que inevitablemente se enfrenta la tecnología y la construcción social de la misma (Latour, Callon 1987). En otras palabras, se trata del contexto social en el que la tecnología se inserta, el uso que hacen los usuarios de ella, y las prácticas de uso que influyen en su desarrollo y diseño. A partir de esto, Sassen (2001) deduce que la ciudad se configura como espacio estratégico para el desarrollo de las nuevas tecnologías, donde un proyecto de ciudad para tener un impacto sobre la dimensión urbana debe desvincularse de las utopías/distopías tecnológicas y afrontar las contradicciones y las problemáticas reales urbanas que pertenecen a la esfera humana y que emergen en forma de necesidades. Sassen (2012) sostiene que "urbanizar las tecnologías" significa hacerlas efectivamente útiles para las nuevas necesidades urbanas.

Construir hipótesis teóricas fundamentadas en la Red de Actores significa no hacer una distinción prioritaria entre actores humanos y no humanos, y colocar a los usuarios, diseñadores y actores políticos dentro de esta red heterogénea. Oudshoorn y Pinch (2008) ofrecen una visión amplia de estudios centrados en las relaciones entre usuarios y tecnologías; abordando el tema de cómo "configurar a los usuarios" y mostrando la importancia de la investigación focalizada en el usuario en base a los Estudios de las Ciencias y Tecnologías. El resultado de éste enfoque es enfatizar cómo la frontera entre el rol del productor y del consumidor está cada vez más distorsionado, y también pueda hablarse a nivel de programación cívica de "usuarios diseñadores", "diseño inteligente" y prosumidor.

\section{COMPARACIÓN DE LA REALIDAD FÍSICA Y LA ESFERA DIGITAL}

El impacto de las tecnologías digitales es considerable y cotidiano en muchos contextos: trabajo, consumo, producción, transporte, movilidad, entretenimiento, relaciones sociales, solo por citar algunos; al punto de tener un profundo impacto en la geografía y en la percepción de la realidad. No existe un punto en el planeta que no se pueda visualizar remotamente a través de "Google Earth", los movimientos de los vehículos pueden ser mapeados (localizados) en tiempo real por dispositivos GPS y controlados por corporaciones que obtienen ventaja directa de la actividad voluntaria de los usuarios. Considérese por ejemplo Waze, el software oficial de los conductores de Uber (propiedad de Google), que permite, a través de la lógica del contenido generado por el usuario (UGC) y una interfaz inspirada en los principios de la gamificación, informar eventos como bloqueo de carreteras, obras viales, embotellamientos y cualquier otra novedad o complicación que pudiera encontrarse en el camino. El software de libre acceso es utilizado también por un gran número de personas que, incluso sin usar los servicios de Uber, contribuyen a generar valor para la empresa multinacional, señalando eventos en las vías o simplemente compartiendo su posición.

Esta participación en la plataforma ofrece al usuario seguramente la ventaja de utilizar un 
servicio de navegación satelital aparentemente gratuito, pero al mismo tiempo enriquece de información al territorio gracias a las publicaciones voluntarias de la comunidad de conductores, lugares, embotellamientos o puntos de control que continúan aumentando gracias a la posibilidad de ser compartidos por las comunidades sucesivas. Una progresión dirigida, por ejemplo, hace que los conductores de Uber puedan, aprovechando los principios de inteligencia colectiva y conectiva (Levy, 2002), prestar su servicio en modo más rápido y eficiente, encontrando caminos menos transitados y vías más rápidas. Una proliferación de datos que sigue una fórmula auto generativa y que representa una de las características fundamentales de una civilización digital, en la cual la frontera entre contenido físico y virtual no solo desaparece sino que se anula el momento en que el territorio, además de la información y su movimiento, pierde su connotación concreta (Masi, 2016).

Se pueden encontrar procesos similares también en otros ámbitos de la economía colaborativa o bajo demanda en el sector residencial, como en AirBnB por ejemplo, con la revolución del mercado de alquiler residencial y vacacional y un mapeo voluntario de todas las propiedades inmobiliarias en el territorio vacante o disponible de alguna manera, por cortos o largos períodos.

Las plataformas digitales, gracias a la participación de los usuarios y con la ayuda de sensores continuamente actualizados, intervienen cambiando progresivamente el concepto mismo de espacio, que es asociado al nivel de interacciones sociales que tienen lugar en este mismo espacio. En otras palabras, cambian la naturaleza del espacio y de las relaciones sociales, la gestión y la gobernanza local, la producción de espacio, la movilidad y el conocimiento espacial. La formación de imaginarios y las políticas locales son cada vez más estrictamente dependientes de la relación con las tecnologías digitales (Elwood, Lezczynskii, 2013; Wilson, 2012; Castells, 1996), como se da en los espacios físicos donde las redes digitales son incorporadas (Murray, 2008), distinguiendo a la vez lo real de lo digital y aprovechando la oportunidad a favor de una realidad aumentada que se enriquece de la big data geolocalizada.

Para comprender mejor la relación que existe entre el espacio físico y el espacio digital, puede ser útil recuperar los conceptos de "sólido" y "fluido" elaborado por Bauman (2000, 2005): el mundo físico, en gran parte sólido, y las realidades más líquidas coexisten en el mundo digital. Parafraseando la concepción weberiana de "jaula de hierro" en relación a la burocracia como estructura organizativa "sólida" y racionalizada, capaz de controlar todos los tipos de movimientos, podemos imaginar las plataformas contemporáneas y las infraestructuras digitales, por su naturaleza líquidas e hiper-racionales, como una "jaula de datos" en la cual, las empresas privadas recogen y archivan cantidades inimaginables de información sobre movimientos, transacciones económicas, consumos y relaciones sociales.

La hipótesis de Bauman según la cual, las estructuras sólidas de consumo continuarán existiendo incluso si están vinculadas a una época pasada, ya que vivimos en una era cada vez más caracterizada por la llamada liquidez, es apoyada también por los más recientes avances en el panorama económico. Colosos del e-commerce global, como Amazon en occidente y Alibaba en oriente que, prácticamente solos monopolizan el sector, están implementando procesos que refuerzan cada vez más su presencia no solamente en el espacio digital, sino sobre todo en el mundo físico, a través de la apertura de puntos de venta y supermercados en el territorio.

La tendencia de combinar la presencia digital con la presencia física en la jerga del marketing se llama brick and click, término que pretende enfatizar la lógica de coexistencia de ambos contextos, en lugar que el dominio de uno sobre el otro, y no solamente en el 
ámbito de consumo, sino también en las interacciones con la ciudad. En este sentido se puede plantear la hipótesis de que el mundo de los átomos, el de la realidad física, se encuentra cada vez más mezclado con el mundo del bit, la realidad digital (Degli Esposti, 2015): "la metrópoli no se anula en las redes virtuales, más bien se transforma a través de la interacción entre la comunicación electrónica y las redes físicas, a través de la combinación de lugar y red" (Castells 2004: p. 57).

En un contexto social y económico en el que los átomos y los bits están cada vez más interconectados (brick and click) se verifican múltiples oportunidades que enfatizan las distintas combinaciones de escasez y abundancia como principios reguladores. La combinación de átomos y bits complica y transforma la dimensión espacio- temporal, el vivir online y offline (en línea y fuera de línea), y la percepción sobre lo que es real y lo que es virtual. Se habla de la transición de una economía basada en átomos, principalmente en el intercambio de bienes físicos, a una basada en un intercambio de bits. El debate sobre átomos y bits lleva a reflexionar acerca de los conceptos de "dualismo digital" y "realidad aumentada" Jurgenson (2012), es decir, sobre la oportunidad de continuar a interpretar la realidad online y offline como dos contextos distintos y separados, o por el contrario, reconocer que entre ellos hay una creciente integración hacia un único contexto emergente.

Es cada vez más evidente que, si bien es aún posible hacer una distinción entre físico y digital, es oportuno pensar en éstos dos conceptos como parte de una nueva realidad aumentada. La vieja ciudad de cemento, vidrio y metal ahora esconde un vasto mundo subterráneo de computadoras y software, aglomeraciones de bases de datos y servidores en constante tensión. Conectados vía internet, estos dispositivos se enlazan en un sistema nervioso que contiene la vida cotidiana de miles de individuos, en un mundo de ciudades enormes y en crecimiento.

Esto representa un cambio histórico en el modo en el que construimos y gestionamos las ciudades. Las ciudades inteligentes son lugares en los que se utiliza la tecnología de la información para afrontar problemas viejos y nuevos. En el pasado, edificios e infraestructuras gestionaban el flujo de personas y mercaderías en formas estrictas y predeterminadas. Sin embargo, las ciudades inteligentes pueden adaptarse rápidamente, extrayendo lecturas de una gran variedad de sensores, y alimentando datos en el software con la capacidad de ver el cuadro general para así poder actuar.

La innovación tecnológica y la difusión de dispositivos y sensores colocan en discusión la noción misma de vigilancia, en cuanto trasforman la relación "observado - observador" y la fusión de los contextos público y privado, ofreciendo la posibilidad de nuevas formas de control, tanto individual como colectivo. En la "ciudad del bit" lo que hacemos es progresivamente rastreable; cada búsqueda que hacemos en internet queda registrada y es utilizada para proporcionarnos ofertas comerciales adaptadas a nuestras preferencias; las publicaciones, las fotos, y las interacciones que colocamos en las redes sociales, son utilizadas para fines comerciales y de control. Cada pago que hacemos con un cajero automático o una tarjeta de débito o crédito, así como cada llamada o producto comprado, son de algún modo atribuibles al perfil que los expertos de marketing han rastreado de nosotros. Nuestras acciones contribuyen a incentivar un proceso de valor en el que a veces, si nos movemos conscientemente, obtenemos beneficios, mientras que en otros casos, sufrimos las consecuencias. En este sentido podemos considerarnos prosumer (prosumidores), habilitados y explotados al mismo tiempo, actores en el proceso de producción y consumo que es principalmente un sistema de control social. Por lo tanto, la vigilancia tiende así a hacerse "líquida" (Bauman, Lyon 2014), y la información obtenida de nuestro cuerpo se convierte en objeto de análisis estadístico a través del cual somos 
encasillados en categorías de consumidores apetecibles o marginales y/o de ciudadanos buenos, malos o "peligrosos".

Según Morozov e Bria (2018), las ciudades inteligentes constituyen ejemplos perfectos de aquello que es llamado corporate storytelling, es decir, narración empresarial, despojados de cada acepción política y aparato crítico. Historias que cuentan la marcha sin freno del progreso y la innovación, vigorosamente acelerada por el ingenio y la inventiva del sector privado, con el objetivo de vender la mayor cantidad de servicios, que a su vez son externalizados y gestionados por infraestructuras privatizadas, para después ofrecer asistencia técnica obligatoria para tales infraestructuras. En este sentido, el corporate storytelling propone un nuevo modelo urbano adherente a la visión neoliberal, en la cual los espacios se perciben sobre todo por su capacidad de generar oportunidades económicas, y las ciudades están controladas por sistemas operativos urbanos respaldados en tres pilares: la posibilidad de estar siempre y en todas partes conectados, las plataformas colaborativas que sostienen la gig economy (economía digital), la difusión de sensores capaces de procesar datos en tiempo real y las plataformas de redes sociales como forma de interacción social dominante (Staffans, Horelli 2004).

Asistimos entonces, a una especie de movimiento cívico en el cual el smartphone (móvil) se convierte al mismo tiempo en una plataforma para reinventar la ciudad desde abajo, y en un medio económico para la resolución de problemas. Experimentamos la simbiosis entre un lugar físico y el ciberespacio que cada día nos dirige hacia ciudades del futuro siempre más inteligentes. Por lo tanto, es casi imposible imaginar la vida de la ciudad sin poder conectarse, sin un kit de supervivencia megaciudad que ayude a buscar, navegar, comunicar y coordinar a todos con todo lo que nos rodea (Goldsmith, 2014). Este proceso no es de ningún modo neutral y libre de riesgos y peligros. Como ha observado Turkle, (2012) los objetos tecnológicos no son solo "meros instrumentos", no existen tecnologías "neutrales", las computadoras son dispositivos tecno-sociales que reconfiguran nociones como los caminos de significado, la identidad, la subjetividad y la conciencia. Las tecnologías digitales corren el riesgo de falsificar nuestras relaciones sociales, ofreciendo a las mismas una réplica sin sentido, una simulación privada de valores esenciales que la caracterizan, produciendo contextos en los que nos encontramos "juntos pero solos". Del mismo modo, Lee y Wellman (2012) hablan de "individualismo conectado" y Castells (2013) de contextos cada vez más dominados por redes "yo céntricas".

\section{CONCLUSIONES: ¿QUÉ CIUDAD INTELIGENTE ES POSIBLE?}

Desde la época de la construcción de las vías y acueductos romanos, ha existido siempre un vínculo entre el desarrollo urbano y la tecnología, que hoy con la tecnología digital, se vuelve aún más fuerte, con un impacto y cambio mucho más rápido e intenso. El debate y la literatura en materia de ciudad inteligente oscila entre dos posiciones dominantes: un enfoque vertical y un enfoque holístico.

El enfoque vertical considera el proyecto de ciudad inteligente esencialmente concentrado en una resolución de problemas específicos de la ciudad, como por ejemplo el tráfico, la contaminación o el consumo de energía, activando diferentes acciones dirigidas a alcanzar objetivos específicos a través del uso de sensores y el análisis de datos asociados a éstos.

Por otro lado, el enfoque holístico, llamado también horizontal, considera la ciudad como un sistema complejo de otros sistemas que interactúan entre sí. La ciudad en este sentido se convierte en una plataforma; una estructura para la detección, comunicación, integración y 
activación de un proceso decisional inteligente. Aún hoy, pese a las grandes inversiones y a la adopción de múltiples soluciones tecnológicas, no existe todavía una ciudad completamente inteligente. El objetivo es desarrollar la ciudad inteligente como una entidad sensible a todos los estímulos ambientales, y responder como un organismo viviente.

Como hemos visto, la ciudad inteligente puede gestionar un círculo virtuoso de feedback $o$ retroalimentación en el cual los proveedores de información suministran los datos necesarios a los usuarios y éstos a su vez tienen la oportunidad de interactuar con los datos de manera que actualizarán y remodelarán el suministro de información, de modo que las nuevas fuentes de datos y los instrumentos de software evolucionen para satisfacer las nuevas exigencias de los usuarios.

Este complejo proceso de triple interacción entre objetos, ciudadanos y relaciones sociales conlleva importantes cuestiones, no solo a nivel de planificación y gestión, sino también sobre qué tipo de modelo tecnológico adoptar, moviéndose hacia la dirección de sistemas propietarios o sistemas abiertos. Compartiendo la opinión de Sassen (2001), hoy las tecnologías digitales no se encuentran lo suficientemente urbanizadas, es decir, no han sido readaptadas a las exigencias específicas de la ciudad y sus habitantes.

El proceso de urbanización de la tecnología consiste en insertar la noción de sistemas inteligentes en la ciudad, transformándola en un laboratorio viviente para las tecnologías urbanas en condición de gestionar todos los principales sistemas que la ciudad tenga necesidad. Durante este proceso se debería activar un debate abierto entre residentes y el liderazgo de la ciudad, destinado a construir un nuevo intercambio de red de código abierto (open source), capaz de relevar y recodificar los problemas en soluciones participativas, proponiendo una dimensión colectiva de actualización y resolución de problemas que involucre también a los ciudadanos, una especie de urbanismo de código abierto.

Otra forma de definir el fenómeno es la propuesta por Morozov y Bria (2018), que introducen el concepto de "soberanía tecnológica", entendida como la capacidad de los ciudadanos de participar y tener voz sobre cómo operan y que objetivos persiguen las infraestructuras tecnológicas que les rodean. Esta idea de soberanía podría estar vinculada a aquella que normalmente se utiliza para la dimensión alimenticia, donde se es propietario de los productos que se consumen, no en el sentido de propiedad, sino en el sentido de la conciencia y del conocimiento de su origen.

La alternativa a un proceso de adopción de tecnologías inteligentes para una ciudad, que no sea el resultado de una urbanización abierta, es estar vinculados a plataformas privadas permaneciendo atrapados en nuevas "jaulas de datos" que generarán nuevas formas de desigualdad, exclusión y estarán sujetas a tasas crecientes de obsolescencia, en las que los sensores se convierten en censores, siguiendo pasivamente el proyecto de los colosos de $\mathrm{Hi}$ Tech de reconstruir el mundo alrededor de nosotros de acuerdo a su visión de eficiencia, seguridad y confort. En la sociedad líquida de la "jaula de datos" las barras no se ven, sino que están incorporadas en las plataformas hardware y software, razón demás para reivindicar que los sistemas digitales reguladores de los procesos en las ciudades sean abiertos, de manera que se permita realmente a las ciudades inteligentes ser de dominio público compartido y tecnológicamente orientado a un desarrollo sostenible.

\section{BIBLIOGRAFÍA}

Ash, J.; Kitchin, R. \& Leszczynski, A. (2018). “Digital turn, digital geographies?” Progress in Human Geography, 42(1): 25-43. DOI: https://doi.org/10.1177/0309132516664800 
Bauman, Z. (2000). Liquid Modernity. Cambridge: Polity Press

Bauman, Z. (2005). Liquid life. Cambridge: Polity Press

Bauman, Z. \& Lyon, D. (2015). Sesto potere: la sorveglianza nella modernità liquida. Bari-Roma: Laterza.

Beck, U. (2000) . La società del rischio. Verso una seconda modernità. Roma: Carocci.

Beer, D. \& Burrows, R. (2010). "Consumption, Prosumption and Participatory Web Cultures An introduction". Journal of Consumer Culture, 10(1): 3-12. DOI: https://doi.org/10.1177/1469540509354009

Bria, F., Morozov, E. (2018). Ripensare la smart city. (Ianelli, F. Trad.) Torino: Codice. (Trabajo original publicado en 2018)

Castells, M. (2004). La città delle reti. (Rizzo, C. Trad.). Venezia: Marsilio.

Castells, M. (2013). Galassia Internet. (Viviani, S. Trad.). Milano: Feltrinelli.

Castells, M. (2014). La nascita della società in rete. (Turquet, L. Trad.). Milano: UBE.

Castrignanò M. (2004). La città degli individui. Tra crisi ed evoluzione del legame sociale, Milano: Franco Angeli.

Cohen B. (2016). The Emergence of the Urban Entrepreneur: How the Growth of Cities and the Sharing Economy Are Driving a New Breed of Innovators, California: ABC-CLIO.

Degli Esposti, P. (2015). Essere prosumer nella società digitale. Produzione e consume tra atomi e bit, Franco Angeli, Milano.

Elwood S. y Leszczynski A. (2013). New Spatial Media, New Knowledge Politics, 38(4): 544-59, DOI: http://dx.doi.org/10.1111/j.1475-5661.2012.00543.x

Giffinger, R.; Fertner, C.; Kramar, H.; Kalasek, R.; Pichler-Milanovic, N. \& Meijers, E. (2007). Smart Cities- Ranking of European medium-sized cities, Vienna: Vienna University of Technology.

Goldsmith S. \& Crawford, S. (2014). The Responsive City Engaging communities through data-smart governance, New Jersey: John Wiley \& Sons.

Howard E. (2017). La Città Giardino del Domani, Milano: Asterios.

Jenkins H. (2006). Convergence Culture: Where Old and New Media Collide, New York: New York University Press.

Jenkins H. (2008). Convergence Culture, New York: NYU Press.

Jurgenson, N. (2012). "When atoms meet bits: Social media, the mobile web and augmented revolution". Future internet, 4(1): 83-91. DOI: https://doi.org/10.3390/fi4010083

Latour, B. (1992). "Where are the missing masses? the sociology of mundane artefacts". W.E.Bijerker \& J. Law. Shaping Technology/Building Society: Studies in Sociotechnical Change, Cambridge: MIT Press, 35: 255-258.

Lee R. y Wellman B. (2012). Networked. Il nuovo sistema operativo sociale, Milano: Guerini \& Associati.

Lévy P. (2002). L'intelligenza Collettiva. Per un'antropologia del cyberspazio, Milano: Feltrinelli. D. Lupton (2015). Digital Sociology, London: Routledge. DOI: https://doi.org/10.4324/9781315776880

Manville, C.; Cochrane, G.; Cave, J.; Millard, J.; Pederson, J. y Thaarup, R. (2014). Mapping Smart Cities in the EU. Parlamento Europeo, Comité de Industria, Investigación y Energía ITRE, accessible en: https://www.europarl.europa.eu/RegData/etudes/etudes/join/2014/507480/IPOLITRE_ET(2014)507480_EN.pdf [consulta 03/01/2020]

Martinotti G. (1993) Metropoli. La nuova morfologia sociale della città, Il Mulino: Bologna.

Mazzette A. y Sgroi M. (2007). La metropoli consumata. Antropologie, architetture politiche, cittadinanze, Milano: Franco Angeli.

Meijer, A. y Bolívar, M.P.R. (2013). Governing the Smart City: Scaling-Up the Search for SocioTechno Synergy. Edinburgh: EGPA.

Ojo, A.; Curry, E.; Janowski, T. \& Dzhusupova, Z. (2015). "Designing Next Generation Smart City Initiatives: The SCID Framework". En Public Administration and Information Technology (8): 4367. Springer. DOI: https://doi.org/10.1007/978-3-319-03167-5 4

Oudshoorn, N. \& Pinch, T. (2008). "User-technology relationships: Some recent developments". The Handbook of Science and Technology Studies, Cambridge: MIT Press, 541-565. 
Pezzini, I. \& Cervelli, P. (2006). Scene del consumo, dallo shopping al museo, Roma: Meltemi.

Pieretti G. y Castrignanò M. (2010). "Consumo di suolo e Urban Sprawl: alcune considerazioni sulla specificità del caso italiano", Sociologia Urbana E Rurale, 92-93.

Ratti C. (2013). Smart City, Smart Citizen, Burago di Molgora MB: EGEA.

Ritzer G. (2014). "Prosumption: Evolution, Revolution, or Eternal Return of the Same?", Journal of Consumer Culture, 14(3): 3-25. DOI: https://doi.org/10.1177/1469540513509641

Rizer G. (2018). The McDonaldization of Society: into the digital age. Thousand Oaks, CA: SAGE Publications

Sartoretti, I. (2012). "Lo sprawl urbano". Micron Urbanistica, 22: 18-23.

Sassen S. (1991). The Global City: New York, London, Tokyo Princeton: Princeton University Press.

Sassen, S. (2001). "Impacts of Information Technologies on Urban Economies and Politics". International Journal of Urban and Regional Research, 25(2): 411-418. DOI: https://doi.org/10.1111/1468-2427.00319

Silverstone R., Haddon, L. (1996). "Design and the domestication of information and communication technologies: technical change and everyday life", Mansell et al. Communication by Design: The Politics of ICTs, Oxford: Oxford University Press, pp. 44-74.

Staffans A. y Horelli L. (2014). "Expanded urban planning as a vehicle for understanding and shaping smart, liveable cities", The Journal of Community Informatics, 10(3).

Townsend A.M. (2013). Smart Cities: Big Data, Civic Hackers, and the Quest for a New Utopia, New York: W.W. Norton \& Company.

Turkle S. (2012). Insieme ma soli. Perché ci aspettiamo sempre più dalla tecnologia e sempre meno dagli altri, Torino: Codice.

Van de Wijdeven, T.; de Graaf, L. y Hendriks, F. (2013). "Actief Burgerschap. Lijnen in de literatuur". Tilburg, Tilburgse School voor Politiek in Bestuur, Amsterdam: Ios Press.

\section{Breve currículo:}

\section{Piergiorgio Degli Esposti}

Profesor titular en la Universidad de Bolonia y uno de los miembros fundadores del Centro de Investigación de Estudios Avanzados Ces.Co.Com que se especializa en Consumo y Comunicación. Desde 2017 es miembro de la Junta Científica de "Sociología del consumo" dentro de la Asociación Europea de Sociología. Su investigación se centra en los comportamientos de consumo digital y la prosumición. Es Coordinador Científico del Laboratorio Multimedia del Departamento de Sociología y Derecho Comercial de la Universidad de Bolonia. Su último libro es Ser un prosumidor en la sociedad digital: producción y consumo entre átomos y bits.

\section{Giovanni Ciofalo}

Profesor titular de sociología de los procesos culturales y comunicativos en la Sapienza Universidad de Roma. Sus principales intereses de investigación se centran en las dinámicas de producción de los medios de comunicación y el consumo cultural, en las teorías y los efectos de la comunicación en la lógica y las prácticas de los medios sociales y el transmedia. Ha publicado artículos y capítulos de libros sobre la evolución de la industria cultural italiana, sobre la relación entre los medios de comunicación, el género y los estereotipos y sobre el impacto cultural y tecnológico de la comunicación. 